\title{
Micobiota do Solo de uma Área de Duna na Restinga da Marambaia, Rio de Janeiro, RJ
}

\author{
Marcelo Elias Fraga ${ }^{1}$, Marcos Gervasio Pereira $^{2}$, Francisco Adriano de Souza ${ }^{3}$ \\ ${ }^{1}$ Núcleo de Pesquisa Micológica e Micotoxicológica, Departamento de Microbiologia e Imunologia Veterinária, \\ Universidade Federal Rural do Rio de Janeiro - UFRRJ \\ ${ }^{2}$ Departamento de Solos, Universidade Federal Rural do Rio de Janeiro - UFRRJ
}

${ }^{3}$ Núcleo de Biologia Aplicada, Embrapa Milho e Sorgo,

\begin{abstract}
RESUMO
Este trabalho avaliou a micobiota do solo de uma duna nos terços inferior, médio e superior de encosta, localizada na Restinga da Marambaia, RJ. A área de estudo com aproximadamente $500 \mathrm{~m}^{2}$ está dividida em três terços, com a vegetação variando de arbustiva a arbórea. Foi delimitada uma gleba de dimensões de 50 x $50 \mathrm{~m}$, onde foram abertas cinco minitrincheiras até a profundidade de $10 \mathrm{~cm}$ para coleta, sendo coletadas três amostras compostas formadas a partir da reunião de cinco amostras simples para análise do solo e caracterização da micobiota. A fertilidade do solo indicou que o terço inferior apresentou maiores teores de alumínio e menores de nutrientes. As unidades formadoras de colônias de fungos variaram de 4,6 x $10^{3}$ a $9 \times 10^{4} \mathrm{ufc}^{-1}$. Quarenta e nove fungos, num total de 85 isolados, pertencentes a 13 gêneros e 32 espécies foram identificados. Os gêneros mais frequentes foram Penicillium, Aspergillus e Trichoderma. A maior riqueza ocorreu no terço médio, porém uma espécie (Trichoderma pseudokoningi) foi comum entre as áreas. Este trabalho sugere que a Restinga da Marambaia apresenta grande diversidade fúngica.
\end{abstract}

Palavras-chave: restinga, micobiota, solo.

\section{Soil Mycobiota in a Dune Area in the Municipality of Restinga da Marambaia, State of Rio de Janeiro, Brazil}

\begin{abstract}
This study was carried out in order to evaluate soil mycobiota in different soil positions (shoulder, back slope, foot slope) in a dune, located in Restinga da Marambaia, RJ. The studied area of approximately $500 \mathrm{~m}^{2}$ was divided in three parts with vegetation ranging from the shrubs to trees. It was a $50 \times 50 \mathrm{~m}$ enclosed plot where five $10 \mathrm{~cm}$-deep mini trenches were opened for soil collection. Three composite samples formed by the gathering of five single samples were collected for analysis and characterization of the soil mycobiota. The highest aluminum content and the lowest nutrient content were observed in the foot slope position. The colony unit formation varied from $4.6 \times 103$ to $9.0 \times 104$ cuf.g $^{-1} .49$ fungi - in a total of 85 isolated, belonging to 13 genders and 32 species - were found. Penicillium, Aspergillus and Trichoderma were the most frequent genders. The highest richness value was observed in the back slope position. Trichoderma pseudokoningi was the most common species found in the areas. This study implies that Restinga da Marambaia presents a great fungal diversity.
\end{abstract}

Keywords: estuarine habitats, mycobiota, soil. 


\section{INTRODUÇÃO}

O solo é um hábitat que oferece ambiente propício ao desenvolvimento microbiano (Moreira et al., 2008). A participação da microbiota do solo no funcionamento, sustentabilidade $e$ produtividade dos ecossistemas é bem reconhecida (Moreira \& Siqueira, 2006), sendo grande o interesse biotecnológico. Além desse fator, alguns parâmetros referentes à diversidade dos microrganismos no solo podem ser utilizados como bioindicadores para avaliação do equilíbrio de ecossistemas florestais (Peña et al., 2005).

Na biosfera, o hábitat mais rico em fungos é o solo. A principal função desses organismos é a degradação de matéria orgânica, envolvendo pelo menos quatro grupos distintos: celulolíticos, hemicelulolíticos, pectinolíticos e ligninolíticos (Tauk, 1990). Além desta função, fungos de solo do filo Glomeromycota estabelecem simbiose mutualística com raízes da maioria das espécies de plantas (Moreira \& Siqueira, 2006).

Dados a respeito das interações microbianas no solo e na rizosfera em ecossistemas tropicais são esparsos, mesmo que a sua importância no equilíbrio ecológico seja incontestável (Abreu \& Pfenning, 2008; Moreira et al., 2008). De acordo com a literatura, pouco se conhece sobre a diversidade fúngica em áreas de restingas (Clipson et al., 2006), principalmente em nosso país, havendo necessidade de estudos mais detalhados sobre esses organismos.

A biodiversidade tem especial relevância quando se refere a ecossistemas reconhecidamente ameaçados. Os ecossistemas litorâneos têm sido historicamente muito descaracterizados ou até mesmo totalmente dizimados, não somente pela facilidade de acesso, como também pela sua beleza natural. No entanto, a Restinga da Marambaia é uma área que se destaca por apresentar florestas de encosta, com elementos típicos de Serra do Mar e um mosaico vegetacional que recobre as planícies arenosas, por dunas de até $30 \mathrm{~m}$ de altura, com cerca de $40 \mathrm{~km}$ de extensão. Essas características estão baseadas fundamentalmente na fisionomia, na composição florística e na saturação hídrica do solo (Menezes \& Araújo, 2005).
O objetivo deste trabalho foi caracterizar a micobiota do solo de uma área de duna na Restinga da Marambaia, RJ.

\section{MATERIAL E MÉTODOS}

A Restinga de Marambaia, com aproximadamente $40 \mathrm{~km}^{2}$, localiza-se no Estado do Rio de Janeiro, compreendendo os Municípios do Rio de Janeiro, Itaguaí e Mangaratiba. A floresta sobre duna localizase na extremidade leste da restinga da Marambaia, Zona Oeste do Município do Rio de Janeiro ( $23^{\circ} 02^{\prime} \mathrm{S}$ e $23^{\circ} 06^{\prime} \mathrm{S} ; 43^{\circ} 35^{\prime} \mathrm{W}$ e $43^{\circ} 54^{\prime} \mathrm{W}$ ). Esta formação vegetal recobre cerca de 527 ha, aproximadamente 10,7\% da área total da Restinga de Marambaia. O clima da região, enquadra-se no macroclima de clima tropical chuvoso. As temperaturas do ar da região são típicas das áreas litorâneas tropicais, com médias mensais sempre acima de $20,0^{\circ} \mathrm{C}$ e a média anual de $23,7^{\circ} \mathrm{C}$. A precipitação média anual é de $1.239,7 \mathrm{~mm}$, e $37 \%$ deste valor ocorrem no verão, distribuídos em torno de 40 dias chuvosos (Mattos, 2005).

A área de estudo com aproximadamente $500 \mathrm{~m}^{2}$ está localizada na Restinga da Marambaia. Foi selecionada uma duna, e esta, dividida em três terços em função do perfil da vegetação que variou de arbustiva, localizada no terço inferior, a arbórea, no terço superior. A gleba de estudo foi nas dimensões de 50 x $50 \mathrm{~m}$. As coletas foram realizadas pela manhã em maio de 2006, sendo as amostras acondicionadas em saco de papel e posteriormente levadas ao laboratório para análise. No momento da coleta, determinou-se a temperatura do solo com geotermômetro digital e tomadas amostras para determinação do conteúdo de água no solo. Em cada gleba, foram abertas cinco minitrincheiras até a profundidade de $10 \mathrm{~cm}$, para coleta de amostras de solo. A coleta foi realizada depois de retirada da serapilheira, na profundidade de $0-5 \mathrm{~cm}$. Para cada terço da duna, foram coletadas três amostras compostas formadas a partir da reunião de cinco amostras simples.

Após a coleta, as amostras foram secas ao ar e posteriormente peneiradas em peneiras de $2,00 \mathrm{~mm}$ de malha. No material obtido foram quantificados os valores de $\mathrm{pH}$ e os teores de $\mathrm{Ca}, \mathrm{Mg}, \mathrm{K}, \mathrm{P}, \mathrm{Na}, \mathrm{Al}$, 
$\mathrm{Al}+\mathrm{H}$ e Carbono orgânico. Todas as análises foram realizadas segundo Embrapa (2006).

O delineamento utilizado foi o inteiramente casualizado. Para os resultados das propriedades químicas, foi feita avaliação da normalidade dos dados (Lilliefors), homogeneidade das variâncias dos erros pelo Teste de Cochran \& Barttlet. Posteriormente, os resultados foram submetidos à análise de variância com aplicação do teste $F$ e os valores médios comparados entre si pelo teste de Tukey a $5 \%$ de probabilidade.

Estes testes e análises foram realizados com o auxílio dos programas estatísticos SAEG-8.0 (Sistema de Análises Estatísticas e Genéticas - Universidade Federal de Viçosa) (Sistema..., 1997) e XLSTAT (2008) para Análise dos Componentes Principais.

A micobiota foi quantificada por unidade formadora de colônia (UFC). Os isolamentos e identificações dos fungos foram feitos em $5 \mathrm{~g}$ de solo ressuspendido em $45 \mathrm{~mL}$ de $0,1 \%$ de peptona contendo $0,1 \%$ de Tween 80 em frasco Erlenmeyer, em seguida agitado por 30 minutos em Shake a $150 \mathrm{rpm}$ (Gomes et al., 2001; Markovina et al., 2005).

A contagem das UFCs de fungos foi realizada segundo metodologia de diluição decimal seriada. Todas as placas foram observadas diariamente, e as placas de meios para enumeração foram selecionadas dentre aquelas que apresentaram número de colônias entre 10 e 100 (Samson et al., 2000). O isolamento foi baseado em meios Agar Batata Dextrose, Agar Aveia e Agar Dicloran Rosa de Bengal Cloranfenicol (DRBC) para as espécies da família Trichocomaceae (Markovina et al., 2005). As identificações das espécies foram em meios específicos para cada grupo de fungo e baseados em estudos morfológicos comparados com bibliografias específicas (Raper \&
Fennell, 1965; Domsch et al., 1983; Klich \& Pitt, 1988; Barnett \& Hunter, 1999; Davet \& Rouxel, 2000; Pitt, 2000; Samson et al., 2000; Klich, 2002; Watanabe, 2002; Samson \& Frisvad, 2004).

\section{RESULTADOS E DISCUSSÃO}

Quanto à fertilidade do solo, verificou-se que os maiores teores de alumínio e os menores de nutrientes ocorreram no terço inferior da duna em estudo. Além disso, observou-se um aumento do conteúdo dos nutrientes nos terços médio e superior da duna.

Não foram observadas significâncias pelo teste $\mathrm{F}$ para os valores de $\mathrm{pH}$, carbono, $\mathrm{H}+\mathrm{Al}, \mathrm{Al}$ e $\mathrm{P}$, sendo que os valores de carbono orgânico estiveram entre 36,33 e 27,67 g. $\mathrm{kg}^{-1} ; \mathrm{H}+\mathrm{Al}$, entre 8,49 e $3,38 \mathrm{cmol}_{c} \cdot \mathrm{kg}^{-1} ; \mathrm{Al}$, entre 0,13 e $0,37 \mathrm{cmol}_{c^{\prime}} \cdot \mathrm{kg}^{-1}$; e $\mathrm{P}$, valores de 2,33 a $4,00 \mathrm{mg} \cdot \mathrm{kg}^{-1}$. De maneira geral, foram verificados maiores valores e $\mathrm{Ca}, \mathrm{Mg}, \mathrm{Na}$ e $\mathrm{K}$, no terço superior da duna (Tabela 1), sendo constado um decréscimo gradual do terço superior para o terço inferior. Os maiores valores destes elementos no terço superior da encosta podem ser decorrentes da adição via efeito aerossol, provocados pelas ondas (Clipson et al., 2006).

Verificou-se uma variação entre as UFCs de fungos nos diferentes terços da duna. Os valores médios encontrados foram $68,3 \times 10^{3}, 61,5 \times 10^{3} \mathrm{e}$ $66,3 \times 10^{3}$ para os terços inferior, médio e superior, respectivamente. Entretanto, foi observada uma diversidade de espécies isoladas de fungos nos diferentes terços da duna. Os fungos saprófitas apresentam-se na sua grande maioria no solo. Entretanto, é extremamente complexa a quantificação

Tabela 1. Teores de Ca, Mg, Na e K nos terços superior, médio e inferior no solo de uma duna localizada na Restinga da Marambaia, RJ.

Table 1. $\mathrm{Ca}, \mathrm{Mg}, \mathrm{Na}$ and $\mathrm{K}$ levels at the shoulder, back slope and foot slope in a dune soil located in Restinga da Marambaia, RJ.

\begin{tabular}{|c|c|c|c|c|}
\hline \multirow[t]{2}{*}{ Área/terços } & $\mathrm{Ca}$ & $\mathrm{Mg}$ & $\mathrm{Na}$ & \multirow{2}{*}{$\frac{\mathrm{K}}{\mathrm{mg} \cdot \mathrm{kg}^{-1}}$} \\
\hline & \multicolumn{3}{|c|}{$\mathrm{cmol}_{\mathrm{c}} \cdot \mathrm{kg}^{-1}$} & \\
\hline Superior & $3,27^{\mathrm{a}}$ & $1,62^{\mathrm{a}}$ & $0,14^{\mathrm{a}}$ & $29,67^{\mathrm{a}}$ \\
\hline Médio & $2,33^{b}$ & $0,87^{b}$ & $0,07^{\mathrm{b}}$ & $20,33^{\mathrm{b}}$ \\
\hline Inferior & $0,45^{\mathrm{b}}$ & $0,67^{\mathrm{c}}$ & $0,06^{\mathrm{b}}$ & $16,00^{\mathrm{b}}$ \\
\hline
\end{tabular}

Letras iguais na coluna não diferem pelo teste de Tukey a $5 \%$ de probabilidade. 
e isolamento deles nesse ambiente (Domsch et al., 1993).

A metodologia de cultivo direto utilizada para caracterização quantitativa da presença de fungos no solo apresentou-se com limitações quando comparada com as técnicas moleculares (Bridge \& Spooner, 2001; Kirk et al., 2004; Vieira \& Nahas, 2005). A grande vantagem do cultivo direto, empregado neste trabalho, em relação à caracterização qualitativa, é que através de cultivo direto é possível isolar fungos, mesmo que estes estejam em pequena quantidade. Favorecendo o isolamento, caracterização, conservação em coleções de cultura e utilização biotecnológica. Quarenta e nove fungos, distribuídos num total de 85 isolados, foram identificados nas nove amostras. Entre os isolados foram identificados 13 gêneros e 32 espécies, e os gêneros que apresentaram as maiores ocorrências foram Penicillium, Aspergillus e Trichoderma (Tabela 2).

A espécie Trichoderma pseudokoningi foi à única espécie encontrada nos três diferentes terço da duna, já as espécies Aspergillus conicus, Aspergillus sydowii e Penicillium miczynskii, em dois terços da duna. Entre os fungos identificados foram encontradas espécies de poucos relatos como Aspergillus citrisporus, Aspergillus conicus, Aspergillus gracilis, Curvularia tuberculata, Gliocladium roseum, Penicillium corylophilum, Penicillium miczynskii, Penicillium arenicola, Penicillium paxilli, Phialophora cyclaminis, Phialophora atrovirens, Pythium echinulatum, Pythium apleroticum, Stachybotrys parvispora, Trichoderma pseudokoningi e Trichoderma aureoviride (Tabela 2).

Verificaram-se valores de $\mathrm{pH}$ variando de 4,84 a 4,91, o que caracteriza as áreas como de $\mathrm{pH}$ ligeiramente ácido, segundo (Deacon, 1997), Pythium spp., geralmente não são tolerantes a valores muito baixos de $\mathrm{pH}$, podendo ocorrer em solos com pH entre 4 e 5, o que foi verificado nas áreas de estudo. Adicionalmente o autor destaca que Trichoderma spp., que também foi encontrado na área da duna, é característico de solos ácidos.

Através da análise de componentes principais (Figuras 1 e 2), observa-se que os elementos $\mathrm{Na}$, $\mathrm{Mg}, \mathrm{K}$ e $\mathrm{H}+\mathrm{Al}$ foram os principais responsáveis pela separação do terço superior da encosta dos

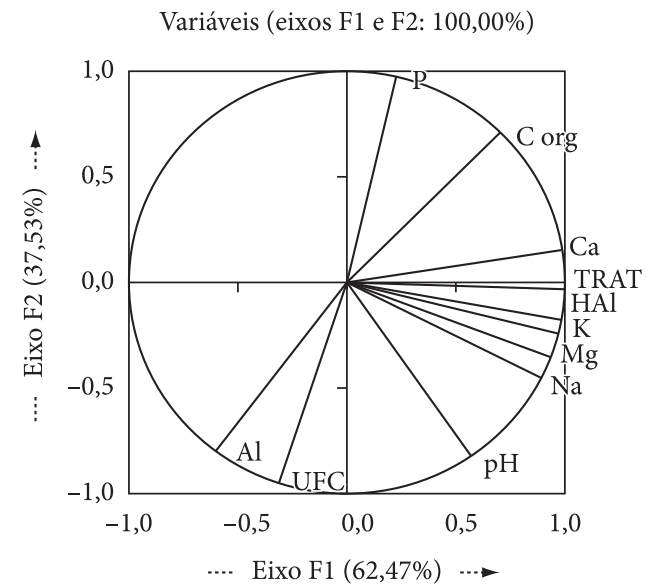

Figura 1. Análise de componentes principais, para as variáveis selecionadas, nos terços inferior, médio e superior da encosta de duna localizada na Restinga da Marambaia, RJ.

Figure 1. Main component analyses for selected variables from shoulder, back slope and foot slope in a dune located in Restinga da Marambaia, RJ.

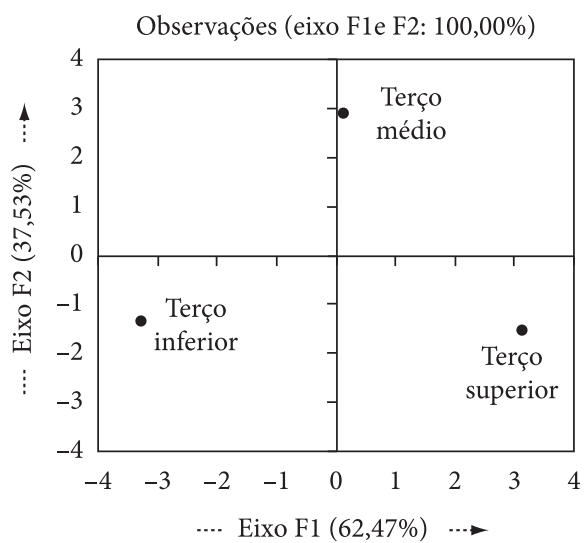

Figura 2. Distribuição das áreas em função das variáveis selecionadas pela análise dos componentes principais.

Figure 2. Distribution areas according to selected variables by main components analyses.

demais terços. Verificou-se também correlação entre as UFCs de fungos e o teor de $\mathrm{Al}(\mathrm{r}=0,98)$, indicando que os maiores valores de alumínio, estão promovendo uma redução no $\mathrm{pH}$ do solo, favorecendo o estabelecimento de um maior número de UFCs de fungos, o que sugere que os principais fungos que compõem essas UFCs de fungos, sejam Pythium spp. e Trichoderma spp.

O conhecimento da micobiota presente na biodiversidade dos solos e sedimentos, de sua 
Tabela 2. Micobiota isolada do solo nos terços inferior, médio e superior da encosta de uma duna localizada na Restinga da Marambaia, RJ.

Table 2. Soil isolated mycobiota from shoulder, back slope and foot slope in a dune located in Restinga da Marambaia, RJ.

\begin{tabular}{llcc}
\multirow{2}{*}{ Fungo } & \multicolumn{3}{c}{ Terço da duna } \\
\cline { 2 - 4 } & Inferior & Médio & Superior
\end{tabular}

Acremonium sp. 1

Acremonium sp. 2

$\mathrm{X}$

A. citrisporus

A. conicus

X

A. gracilis

$\mathrm{x}$

A. niger

A. parasiticus

$\mathrm{x}$

A. sojae

A. sydowii

A. tamarii

$x$
$x$

Curvularia brachyspora

C. clavata

C. tuberculata

Cladosporium cladosporioides

Fungo hialino 1

Fungo hialino 2

Fungo hialino 3

Fungo hialino 4

Fungo hialino 5

Fungo hialino 6

$\mathrm{X}$

Fungo hialino 7

Fungo hialino 8

$\mathrm{X}$

Fungo dematiáceo 1

Fungo dematiáceo 2

Gliocladium sp.

G. roseum

Mucor sp. 1

Mucor sp. 2

Mortierella sp. 1

$\mathrm{x}$

Mortierella sp. 2

Penicillium sp. 1

$\mathrm{x}$

$\mathrm{x}$

$\mathrm{x}$

Penicillium sp. 2

P. arenicola

P. corylophilum

P. decumbens

P. echinulatum

P. javanicum

P. miczynskii

P. paxilli

P. purpurogenum

$\mathrm{X}$

P. sclerotiorum

$\mathrm{X}$

P. simplicissimum

Phialophora sp.

$\mathrm{X}$

P. atrovirens 
Tabela 2. Continuação...

Table 2. Continued...

\begin{tabular}{|c|c|c|c|}
\hline \multirow{2}{*}{ Fungo } & \multicolumn{3}{|c|}{ Terço da duna } \\
\hline & Inferior & Médio & Superior \\
\hline P. cyclaminis & & $\mathrm{X}$ & \\
\hline Phoma sp. 1 & $\mathrm{x}$ & & \\
\hline Phoma sp. 2 & & & $\mathrm{x}$ \\
\hline Pythium sp. 1 & & $\mathrm{X}$ & \\
\hline Pythium sp. 2 & & & $\mathrm{x}$ \\
\hline P. apleroticum & & $\mathrm{X}$ & \\
\hline P. echinulatum & & $\mathrm{X}$ & \\
\hline Stachybotrys parvispora & & $\mathrm{X}$ & \\
\hline Trichoderma aureoviride & $\mathrm{x}$ & & \\
\hline T. harzianum & & $\mathrm{X}$ & \\
\hline T. pseudokoningi & $\mathrm{x}$ & $\mathrm{X}$ & $\mathrm{x}$ \\
\hline Espécies endêmicas & 14 & 20 & 18 \\
\hline Total & 17 & 23 & 20 \\
\hline
\end{tabular}

interação e taxas de ocorrência nos diferentes biomas, sob diversas pressões antrópicas, é bastante limitada em virtude da escassez de estudos taxonômicos disponíveis. Assim, torna-se necessário explorar e preservar essa biodiversidade, o que resultará em benefícios futuros. Assim, torna-se necessário também obter informações científicas sobre métodos padrão para avaliar e monitorar a biodiversidade desses solos e sedimentos, e realizar experimentos quantitativos e qualitativos a fim de entender o papel dos fungos de solo e de sedimentos no funcionamento do ecossistema.

\section{CONCLUSÕES}

Os gêneros mais frequentes encontrados nas áreas de duna na Restinga da Marambaia foram Penicillium, Aspergillus e Trichoderma. A maior riqueza ocorreu no terço médio com 23 espécies, seguida do terço superior com 20 e terço inferior com 17. Uma espécie (Trichoderma pseudokoningi), porém, foi comum entre as áreas, sugerindo que a restinga apresenta grande diversidade fúngica.

\section{AGRADECIMENTO}

Ao Prof. Luis Fernando Tavares de Menezes, que nos proporcionou a coleta e nos incentivou a realizar o presente estudo.

\section{STATUS DA SUBMISSÃO}

Recebido: 13/11/2008

\section{AUTOR(ES) PARA CORRESPONDÊNCIA}

\section{Marcelo Elias Fraga}

Núcleo de Pesquisa Micológica e

Micotoxicológica,

Departamento de Microbiologia e Imunologia

Veterinária,

Universidade Federal Rural do Rio de Janeiro,

BR 465, Km 07, CEP 23890-000,

Seropédica, RJ, Brasil

e-mail:fraga@ufrrj.br

\section{REFERÊNCIAS}

Abreu LM, Pfenning LH. Diversidade de microfungos em solos tropicais. In: Moreira MS, Siqueira JO, Brussaard L, editors. Biodiversidade do solo em ecossistemas brasileiros. Lavras, MG: Ed.UFLA; 2008. p. $444-481$.

Barnett HL, Hunter BB. Illustrated genera of imperfect fungi. $4^{\text {th }}$ ed. Saint Paul, MN: APS Press; 1999.

Bridge P, Spooner B. Soil fungi: diversity and detection. Plant and Soil 2001; 232:147-154. doi:10.1023/A:1010346305799

Clipson N, Otte M, Landy E. Biogeochmical roles of fungi in marine and estuarine habitats. In: Gadd 
GM. Fungi biogeochemical cycles. Cambridge, UK: Cambridge University Press; 2006. p. 437-461. doi:10.1017/CBO9780511550522.019

Davet P, Rouxel F. Detection and isolation of soil fungi. Plymouth, UK: INRA Science Publishers; 2000.

Deacon JW. Modern mycology. Berlin, Germany: Blacwell Science; 1997.

Domsch KH, Gams W, Anderson TH. Compedium of soil fungi. London: Academic Press; 1993. 860 p.

Embrapa, CNPS. Sistema Brasileiro de classificação de solos. 2. ed. Rio de Janeiro: EMBRAPA Solos; 2006.

Gomes NCM, Heuer H, Schonfeld J, Costa R, Mendonça-Hagler L, Smalla K. Bacterial diversity of the rhizosphere of maize (Zea Mays) grow in tropical soil studied by temperature gradient gel eletrophoresis. Plant and Soil 2001; 232:167-180. doi:10.1023/A:1010350406708

Kirk JL, Beaudette LA, Hart M, Moutoglis P, Klironomos JN, Lee $\mathrm{H}$ et al. Methods of studying soil microbial diversity. Journal of Microbiological Methods 2004; 58:169-188. PMid:15234515. doi:10.1016/j. mimet.2004.04.006

Klich MA, Pitt JI. A laboratory guide to common Aspergillus species and their teleomorphs. North Ryde, Australia: CSIRO, 1988. 116 p.

Klich MA. Identification of common Aspergillus species. Utrecht, Netherlands: CBS; 2002.

Markovina A, Pitt JI, Hocking AD, Carter DA, McGee PA. Diversity of the Trichocomaceae in the Katandra Nature Reserve, Central Coast, NSW, Australia. Mycological research 2005; 109:964-973. PMid:16209302. doi:10.1017/S0953756205003540

Mattos CLV. Caracterização climática da Restinga da Marambaia. In: Menezes LFT, Peixoto AL, Araujo DSD, editors. Historia natural da Marambaia. Seropédica, RJ: EDUR.; 2005. p. 55-66.
Menezes LFT, Araujo DSD. Formações vegetais da Restinga da Marambaia, Rio de Janeiro. In: Menezes LFT, Peixoto AL, Araujo DSD, editors. Historia natural da Marambaia. Seropédica, RJ: EDUR; 2005. p. 67-120.

Moreira MS, Siqueira JO, Brussaard L. Biodiversidade do solo em ecossistemas brasileiros. Lavras, MG: Ed.UFLA; 2008 .

Moreira MS, Siqueira JO. Microbiologia e bioquímica do solo. Lavras, MG: Ed.UFLA; 2006.

Peña MLP, Marques R, Jahnel MC, Anjos A. Respiração microbiana como indicadores da qualidade do solo em ecossistema florestal. Floresta 2005; 35(1):117-126.

Pitt JI. A laboratory guide to common Penicillium species. Australia: Food Science Australia a Joint Venture of CSIRO and AFISC; 2000.

Raper KB, Fennell DI. The genus Aspergillus. Baltimore: Williams \& Wilkins; 1965. 686 p.

Samson RA, Frisvad JC. Penicillium subgenus Penicillium: new taxonomic schemes, mycotoxins and other extrolites. Studies in Mycology 2004; 49: 1-251.

Samson RA, Hoekstra ES, Frisvad JC, Filtenborg O. Introduction to food and airborne fungi. $6^{\text {th }}$ ed. Baarn, The Netherlands: CBS; 2000.

Sistemas de Análises Estatísticas e Genéticas - SAEG [CD-ROM]. Viçosa, MG: Universidade Federal de Viçosa; 1997. Versão 8.0.

Tauk SM. Biodegradação de resíduos orgânicos no solo. Revista Brasileira de Geociências 1990; 20(1-4):299-301.

Vieira FCS, Nahas E. Comparison of microbiol numbers in soils by using various culture media and temperatures. Microbiological Research; 2005; 160:197-202. PMid:15881837. doi:10.1016/j.micres.2005.01.004

Watanabe T. Pictorial atlas of soil and seed fungi morphologies of cultured fungi and key to species. $2^{\text {nd }}$ ed. Londom, UK: CRC Press; 2002.

XLSTAT Statistical Analysis [online]. [cited 2008 set. 10]. Available from: http://www.xlstat.com. 